OPEN ACCESS

Edited by:

Keqiang Wu,

National Taiwan University, Taiwan

Reviewed by:

He Huang,

Donald Danforth Plant Science

Center, USA

Ming LuO,

South China Botanical Garden,

Chinese Academy of Sciences, China

*Correspondence:

Alexandre L. Nepomuceno

alexandre.nepomuceno@embrapa.br

Specialty section:

This article was submitted to

Plant Genetics and Genomics,

a section of the journa

Frontiers in Plant Science

Received: 23 January 2017

Accepted: 06 April 2017

Published: 20 April 2017

Citation:

Marcolino-Gomes J, Nakayama TJ,

Molinari HBC, Basso MF

Henning LMM, Fuganti-Pagliarini $R$, Harmon FG and Nepomuceno AL

(2017) Functional Characterization

of a Putative Glycine max ELF4

in Transgenic Arabidopsis and Its Role

during Flowering Control.

Front. Plant Sci. 8:618.

doi: 10.3389/fpls.2017.00618

\title{
Functional Characterization of a Putative Glycine max ELF4 in Transgenic Arabidopsis and Its Role during Flowering Control
}

\begin{abstract}
Juliana Marcolino-Gomes ${ }^{1}$, Thiago J. Nakayama², Hugo B. C. Molinari², Marcos F. Basso ${ }^{2}$, Liliane M. M. Henning ${ }^{1}$, Renata Fuganti-Pagliarini', Frank G. Harmon ${ }^{3,4}$ and Alexandre L. Nepomuceno ${ }^{1 *}$
\end{abstract}

${ }^{1}$ Embrapa Soybean, Brazilian Agricultural Research Corporation, Londrina, Brazil, ${ }^{2}$ Embrapa Agroenergy, Brazilian Agricultural Research Corporation, Brasilia, Brazil, ${ }^{3}$ Plant Gene Expression Center, Agricultural Research Service - United States Department of Agriculture, Albany, NY, USA, ${ }^{4}$ Department of Plant and Microbial Biology, University of California-Berkeley, Berkeley, CA, USA

Flowering is an important trait in major crops like soybean due to its direct relation to grain production. The circadian clock mediates the perception of seasonal changes in day length and temperature to modulate flowering time. The circadian clock gene EARLY FLOWERING 4 (ELF4) was identified in Arabidopsis thaliana and is believed to play a key role in the integration of photoperiod, circadian regulation, and flowering. The molecular circuitry that comprises the circadian clock and flowering control in soybeans is just beginning to be understood. To date, insufficient information regarding the soybean negative flowering regulators exist, and the biological function of the soybean ELF4 (GmELF4) remains unknown. Here, we investigate the ELF4 family members in soybean and functionally characterize a GmELF4 homologous gene. The constitutive overexpression of GmELF4 delayed flowering in Arabidopsis, showing the ELF4 functional conservation among plants as part of the flowering control machinery. We also show that GmELF4 alters the expression of Arabidopsis key flowering time genes (AtCO and AtFT), and this down-regulation is the likely cause of flowering delay phenotypes. Furthermore, we identified the GmELF4 network genes to infer the participation of GmELF4 in soybeans. The data generated in this study provide original insights for comprehending the role of the soybean circadian clock ELF4 gene as a negative flowering controller.

Keywords: A. thaliana, circadian clock, flowering time, gene expression, overexpression, soybean, transgenic

\section{INTRODUCTION}

Soybean (Glycine max L. Merrill) is a globally important leguminous crop that produces highquality protein and oil used in human and animal feed and has potential for biofuels. Sustainable intensification of crop production is crucial for meeting the food demand of the growing human population (Godfray et al., 2010). Flowering is directly related to seed production and, thus, to grain productivity. Hence, understanding the genes and the molecular network that control flowering is of great interest to improve crop productivity. 
The circadian clock is a molecular oscillator that controls fundamental aspects of plant development and metabolism, from gene expression and protein catabolism to control of leaf position and flowering (McClung, 2011). It is believed to enhance fitness by synchronizing the metabolic and physiological processes to the different periods of the day and is considered an important adaptive characteristic (Yerushalmi and Green, 2009). The plant circadian clock interprets seasonal changes in day length and temperature in order to modulate flowering time, ensuring that the transition from vegetative to reproductive stages occurs under optimal physiological and environmental conditions, enhancing reproductive success (Adams and Carré, 2011).

The most complete existing model of the circadian clock in plants is described for Arabidopsis (Arabidopsis thaliana) and is composed of interlocked feedback loops, which includes a loop of three sequential negative steps: (a) the inhibition of evening complex (EC) genes (ELF3, LUX, and ELF4) (Nusinow et al., 2011) by the increase of LHY/CCA1 during the late night, (b) the inhibition of PRR genes by the EC during the early night, and (c) the inhibition of LHY/CCA1 by PRRs during the day (Pokhilko et al., 2012). In this model, the two partially redundant morning MYB-like transcription factors CCA1 and LHY reach peak levels in the morning to repress the daytime expression of TOC1 and GI (Harmer et al., 2000; Alabadí et al., 2001). It was also demonstrated that the inhibitory action of LHY/CCA1 extends to all evening genes (TOC1, LUX, ELF4, ELF3, and GI) (Pokhilko et al., 2012). In turn, TOC1 appears to repress CCA1 and LHY expression within the morning loop (Gendron et al., 2012; Pokhilko et al., 2012), while the EC indirectly activates CCA1/LHY expression by suppressing the expression of CCA1/LHY's repressors (Kikis et al., 2005; Dixon et al., 2011; Helfer et al., 2011; Pokhilko et al., 2012). On the other hand, GI increases TOC1 expression by the inhibition of the EC, which is a negative regulator of TOC1 expression (Pokhilko et al., 2012). Together, the three interlocking feedback loops ensure that the clock produces robust and accurate rhythms necessary to control plant physiological and developmental processes, among them, flowering time.

The photoperiodic-dependent regulation of flowering also has been covered in detail in Arabidopsis, a facultative longday plant [the floral transition occurs earlier when plants are grown under long days (LD) than when they are grown under short days $(\mathrm{SD})]$. In this plant, the perception of day length and flowering is mainly coordinated by the transcription factor CONSTANS (CO), and the expression of CO is finely tuned by the circadian clock (Wenkel et al., 2006). Under SD conditions, the expression of the CO mRNA occurs after dark, with RNA levels peaking $16 \mathrm{~h}$ after dawn, and the $\mathrm{CO}$ protein is rapidly degraded by the action of COP1 and the proteasome, resulting in flowering inhibition (Jang et al., 2008). Conversely, under LD conditions, the peak of $\mathrm{CO}$ expression occurs during daylight, when the activity of COP 1 is inhibited, resulting in CO protein accumulation, and, consequently, flowering induction by the activation of the floral integrators FLOWERING TIME (FT) and TWIN SISTER OF FT (TSF) (Yamaguchi et al., 2005; Jang et al., 2008). In addition, the F-box protein and blue light photoreceptor FKF1 and GI act synergistically to promote the degradation of CDF1, a transcriptional repressor, enabling $C O$ expression at the end of a long day (Sawa et al., 2007). The product of $F T$ expression migrates to the apical meristem, where it initiates the transcriptional reprogramming that results in flowering (Turnbull, 2011).

The circadian clock gene EARLY FLOWERING 4 (ELF4) is believed to play a key role in the integration of photoperiod perception, circadian regulation, and flowering (Doyle et al., 2002). The ELF4 gene was identified through reverse genetic screens for flowering time mutants. The elf4 mutants have elongated hypocotyls and petioles, flower early and lack the ability to sense day length. The early flowering behavior of elf4 mutants is accompanied by misregulation of the flowering activator CO (Doyle et al., 2002). Molecular analysis data reveal ELF4 sequesters GI from the nucleoplasm by a physical interaction, impeding GI to bind at the promoter of $C O$, altering flowering time (Kim et al., 2013).

ELF4 is essential for the maintenance of circadian rhythms, since the elf4 mutation disrupts rhythmic CCA1 expression (Doyle et al., 2002). It is also known that ELF4, together with TOC1, plays a major role in phytochrome B-mediated input to the clock (red light perception) (Kikis et al., 2005). The Arabidopsis ELF4 protein has 111 amino acids and does not contain any known protein signatures (Doyle et al., 2002). This protein belongs to the DUF1313 family, which is a family of proteins that share a domain of unknown function exclusively found in plants (DUF1313).

In contrast to the large volume of data on flowering in LD plants, in SD plants, such as rice and soybeans, the molecular basis of flowering control is still being identified and characterized. Most soybean cultivars have a SD requirement for floral induction: flowering remains suppressed under LD conditions and is induced when the day length is shorter than a critical length, and this sensitivity to photoperiod varies among cultivars (cultivars adapted to high latitudes have weak or absent sensibility to photoperiodic changes) (Watanabe et al., 2012).

There are at least 10 FT genes in soybean, arranged in five sets of gene pairs linked in tandem (Kong et al., 2010). Many studies on the photoperiod response indicate that FT and its homologes are universal signaling molecules for flowering plants (Turnbull, 2011). Furthermore, 26 genes orthologous to the CO gene of Arabidopsis were identified in soybean, named G. max CONSTANS-Like ( $\mathrm{GmCOL}$ ). Gene expression analysis revealed that in SD conditions GmCOL1a and GmCOL1b mRNA peaks occur at the end of the morning and coincide with the peak of the soybean FT orthologous gene, GmFT5a, inducing flowering. On the other hand, under LD, the expression of GmCOL1a and $G m C O L 1 b$ occurs during the night and declines before dawn, and in those conditions the expression of GmFT5a is not induced (Wu et al., 2014).

Although some information about soybean flowering induction genes (FT and CO) exists, at the moment, there is no information regarding soybean negative flowering regulators, such as ELF4. In a previous study, we identified a GmELF4 candidate gene and showed its daily expression pattern of mRNA (Marcolino-Gomes et al., 2014). In this context, here we identify and characterize soybean ELF4 genes and show how a 
GmELF4 homolog alters the expression of key flowering genes in transgenic Arabidopsis, resulting in flowering delay.

\section{MATERIALS AND METHODS}

\section{Identification, Phylogenetic Relationships, and Protein Domain Analysis of ELF4 Members in Soybean and Other Plants}

For the identification of putative soybean ELF4 genes and proteins, the corresponding Arabidopsis ELF4 protein sequence (encoded by AT2G40080) was used as a query in BLAST searches (TBLASTN tool) (Altschul et al., 1990) of the G. max genome version Wm82.a2.v1, available from the Phytozome database ${ }^{1}$, using a cutoff Expect value (E-value) of 1E-12. The soybean ELF4 genes and proteins are representative of the respective gene model 'Glymas.' The ELF4 homologous proteins in plant species were searched for in the NCBI protein database ${ }^{2}$, using keyword searches and are represented by their respective GenInfo Identifier number (GI number).

Phylogenetic relationships between the soybean and other plant ELF4 proteins were considered. For this purpose, after removing the redundancy, the amino acid sequences were subjected to global alignment using the ClustalW algorithm from the Molecular Evolutionary Genetics Analysis version 5.0 (MEGA 5) software package (Tamura et al., 2011), and a phylogenetic tree was constructed using the neighbor-joining (NJ) method with the following parameters: Poisson correction, pairwise deletion and bootstrap (1000 replicates; random seed). Given that the functional domains have importance for the function and classification of ELF4 proteins, the conserved amino acid domains of the ELF4 proteins were analyzed using the motif discovery tool MEME program version 4.11.2 ${ }^{3}$.

\section{ELF4 Soybean Paralogous Genes' Expression}

In a previous study, we identified a GmELF4 candidate gene Glyma.18G027500 (former Glyma18g03130) and showed its daily expression pattern of mRNA using RT-qPCR (Marcolino-Gomes et al., 2014). In the present study, the gene expression patterns of all ELF4 soybean paralogous genes were assessed through analysis of an RNA-Seq database ${ }^{4}$, built from soybean plants of the BR16 genotype (Rodrigues et al., 2015). Plants were grown in $14 \mathrm{~h} \mathrm{light} / 10 \mathrm{~h}$ dark cycles, with $500 \mu \mathrm{mol} \mathrm{m}^{-2} \mathrm{~s}^{-1}$ of white light (provided by cool white fluorescent bulbs), under $28^{\circ} \mathrm{C} / 20^{\circ} \mathrm{C}$ temperature cycles during the light and the dark periods, respectively. Sampling was performed 15 days after germination, when the plants reached the V2 developmental stage (Fehr et al., 1971), at which fully expanded V1 leaves were collected from the three plants at 4-h intervals from the time the lights came on [Zeitgeiber time (ZT) 0].

\footnotetext{
${ }^{1}$ http://phytozome.net/soybean

${ }^{2}$ http://www.ncbi.nlm.nih.gov/protein

${ }^{3} \mathrm{http}: / /$ meme-suite.org/tools/meme

${ }^{4} \mathrm{http}: / /$ www.ncbi.nlm.nih.gov/geo/query/acc.cgi?acc=GSE69469
}

Total RNA was extracted from leaves using the RNA Plant Reagent ${ }^{\circledast}$ according to the manufacturer's instructions (Ambion, Austin, TX, USA) and treated with DNAse (Invitrogen, Carlsbad, CA, USA). High-quality total RNA (RIN $\geq 8.0$ ) was used to analyze the transcripts for each time point: ZT0, $4,8,12,16$, and 20. Equimolar quantities of purified total RNA samples from two independent biological replicates were pooled into one template for library synthesis. For each time period/treatment, three independent libraries were synthesized. The RNA-Seq libraries were built using the NuGEN OvationH kit according to the manufacturer's instructions (NuGEN Technologies, Inc., San Carlos, CA, USA). The libraries obtained were subjected to sequencing by an Illumina HiSeq 2000 system (Illumina, San Diego, CA, USA), using the pairedend mode. Mapping of the reads was performed with the soybean genome (Phytozome Glycine max version 1.1) using the GeneSifter platform ${ }^{5}$. To compare gene expression profiles between different times and treatments (control and drought stress), we $\log _{2}$-transformed the normalized reads [mapped reads per million (RPM)]. The gene expression data obtained at the RNA-seq analyses was validated by qPCR (Rodrigues et al., 2015).

\section{Overexpression of GmELF4 in Arabidopsis}

The coding sequence (CDS) of the GmELF4 gene (Glyma.18G027500) was amplified from genomic DNA from soybean (genotype Williams 82) by PCR reactions using the specific 5'-CACCATGGAAGACCCCTC- ${ }^{\prime}$ and $5^{\prime}$-TTATTTGG AGGAGTTTTTGGAG-3' forward and reverse primers, respectively, and the Platinum ${ }^{\circledR}$ Taq DNA Polymerase High Fidelity (ThermoFisher Scientific, Waltham, MA, USA). The sequence $5^{\prime}$-CACC- $3^{\prime}$ was added to the $5^{\prime}$ upstream region of the forward primer to ensure directional subcloning of the CDS with the $\mathrm{pENTR}^{\mathrm{TM}} / \mathrm{D}-\mathrm{TOPO}^{\circledR}$ (ThermoFisher Scientific, Waltham, MA, USA) entry vector. After confirmation by sequencing, the GmELF4 CDS was transferred to the destination overexpression vector $\mathrm{p}^{*} 7 \mathrm{WG} 2 \mathrm{D}$ (Karimi et al., 2002) using the Invitrogen ${ }^{\mathrm{TM}}$ Gateway ${ }^{\mathrm{TM}}$ recombination cloning system (ThermoFisher Scientific, Waltham, MA, USA). With the $\mathrm{p}^{*} 7 \mathrm{WG} 2 \mathrm{D}$ binary vector, the GmELF4 CDS is constitutively expressed due the effect of the Cauliflower mosaic virus $35 S$ (CaMV35S) promoter. In addition, the construct cassette harbors a rolD promoter fused to the CDS of the enhanced green-fluorescent protein (GFP) linked to an endoplasmic reticulum-targeting signal (pRolD-EgfpER-T35S), used as a fluorescence marker (Karimi et al., 2002).

The resultant gene construct was used for Arabidopsis thaliana ecotype Columbia (Col-0) transformation mediated by Agrobacterium tumefaciens (strain GV 3101) using the floral dip method (Zhang et al., 2006). Seeds were screened in $1 \%$ agar containing Murashige and Skoog (MS) medium containing $15 \mu \mathrm{g} \mathrm{mL}{ }^{-1}$ hygromycin $\mathrm{B}$, as described by Harrison et al. (2006). Hygromycin-resistant plants were confirmed via PCR using specific primers for the gene construct (described above).

\footnotetext{
${ }^{5} \mathrm{http}: / /$ www.geospiza.com/Products/AnalysisEdition.shtml
} 
Since the selection of transgenic lines with low T-DNA copy number is advised to prevent transgene silencing, in our study, we carefully evaluated the T-DNA segregation during three generations, selecting lines that presented Mendelian inheritance for a single characteristic [three transformed plants: one wildtype (WT); 3:1], which would carry just one copy of the transgene. Three independent T3 homozygous lines containing a single copy of the transgene were obtained for further analysis.

\section{Transgenic Plants Phenotype}

The development of plants from three transgenic lines was monitored in growth chambers with controlled temperature $\left(22 \pm 2{ }^{\circ} \mathrm{C}\right)$ and relative humidity $(50 \pm 10 \%)$. Plants were cultivated under LD and SD conditions, with 16 and $10 \mathrm{~h}$ of light, respectively. After plant emergence, the rosette leaf numbers, flowering time and progression of silique formation were monitored weekly. Each line was evaluated via 10 biological replicates. Transgenic plants developmental features were compared to those of non-transformed plants (WT) in both photoperiodic conditions.

\section{GFP Detection in Transgenic Plants}

Green-fluorescent protein fluorescence was evaluated in leaves and roots of 5-day-old plants. Fresh tissue was analyzed with an Axio Scope A1 microscope (Zeiss, Oberkochen, Germany) equipped with a GFP filter (Zeiss filter set 38; Zeiss) coupled to a charge-coupled device (CCD) camera (Motic, Causeway Bay, Hong Kong) for image acquisition Image was captured using Motic Image Plus version 2.0 software (Motic, Causeway Bay, Hong Kong) with the following parameters: exposure $=500$, gain $=2.38$, gamma $=0.10 / 191$ and size $=2592 \times 1944$ pixels. The image was magnified by a factor of $100 \mathrm{x}$, which corresponds to the product of the objective magnification (10x) and the eyepiece lens (10x).

Image analysis for GFP quantitation was performed with Image J software version $1.51 \mathrm{~h}^{6}$. In this analysis, images were converted to 32-bit type and the mean gray values were measured using the ROI analysis tool. The mean gray values of WT plants were used for background normalization of GFP intensity in transgenic plants, generating the relative intensity values. A total of 30 independent sample areas were analyzed in each image.

\section{Gene Expression Analysis via RT-qPCR}

The overexpression of GmELF4 (Glyma.18G027500) and the expression of the Arabidopsis flowering genes AtFT (AT1G65480) and AtCO (AT5G15840) were evaluated in transgenic Arabidopsis via RT-qPCR assays.

Primers for GmELF4 (forward 5'-TGATTCAGCAGGTGAA CGAG-3' and reverse 5'-GACAACCTTGGAGATGTTGC-3') were designed with the Primer3 Plus online tool (Rozen and Skaletsky, 1999) ${ }^{7}$, based on the Glyma.18G027500.1 transcript sequence $^{8}$. The Arabidopsis AtCO and AtFT primer sequences were retrieved from Ito et al. (2012).

\footnotetext{
${ }^{6}$ https://imagej.nih.gov/ij/download.html

${ }^{7}$ http://www.bioinformatics.nl/cgi-bin/primer3plus/primer3plus.cgi

${ }^{8}$ http://www.phytozome.net/search.php? method=Org_Gmax
}

Gene expression was evaluated in rosette leaves of three independent transgenic lines and in WT plants, cultivated in LD and SD conditions, with 16 and $10 \mathrm{~h}$ of light, respectively. For RNA extraction, each replicate tissue was ground to a fine powder in liquid nitrogen, and total RNA was isolated using TRIzol reagent (Invitrogen), according to the manufacturer's instructions. Contaminating DNA in the total RNA was removed using a TURBO DNA-free Kit, according to the manufacturer's instructions (Life Technologies, Grand Island, NY, USA). High-quality total mRNA was used to synthesize cDNA strands (Superscript III First Strand Synthesis, Invitrogen/Life Technologies, Grand Island, NY, USA). The quality of the cDNA and contamination with genomic DNA were examined using a standard PCR assay with primers that spanned an intronic region of the $\beta$-ACTIN Arabidopsis gene (AT5G09810). High-quality cDNA was used to analyze the transcripts in each treatment.

RT-qPCR amplifications were performed in a 7300 RT-qPCR Thermocycler (Applied Biosystems/Life Technologies, Grand Island, NY, USA) with the following cycling parameters: $50^{\circ} \mathrm{C}$ for $2 \mathrm{~min}$; $95^{\circ} \mathrm{C}$ for $10 \mathrm{~min}$; and 45 cycles of $95^{\circ} \mathrm{C}$ for $2 \mathrm{~min}$, $60^{\circ} \mathrm{C}$ for $30 \mathrm{~s}$ and $72^{\circ} \mathrm{C}$ for $30 \mathrm{~s}$. Standard curves were produced from serial dilutions of a cDNA pool to estimate the efficiency of the PCR amplification with each pair of primers. The primer concentrations were adjusted to achieve efficiency rates higher than $85 \%$.

After carrying out the efficiency analysis, the expression levels of the transgene (GmELF4) and flowering genes (AtFT and $A t C O)$ were analyzed separately in transgenic and WT plants, cultivated in $\mathrm{LD}$ and SD conditions. The reactions were performed in triplicate, with cycling parameters similar to those described above for the amplification efficiency analysis. Expression of target genes was normalized using the Arabidopsis endogenous gene PP2A (AT1G13320) (forward primer 5'-ACGT GGCCAAAATGATGCAA- $3^{\prime}$ and reverse primer $5^{\prime}$-TCATGTTC TCCACAACCGCT- $3^{\prime}$ ). The flowering genes (AtFT and AtCO) relative expression was calibrated using WT plants grown under the same environmental conditions. The gene expression analysis was performed using the Rest2009 software package version 2.0.13 (Pfaffl et al., 2002).

\section{The GmELF4 Network Genes}

The GmELF4 gene co-expression regulatory network was evaluated with the Soybean Functional Genomics Database (SFGD) platform (Yu et al., 2014). The SFGD database contains microarray gene expression profiles of 255 samples from experiments of 14 groups and mRNA-Seq data of 30 samples from experiments of four groups, including spatial and temporal transcriptome data for different soybean developmental stages and environmental stresses, providing a robust database for functional analysis of genes of interest. This platform is publically accessible ${ }^{9}$. Pearson's correlation coefficients (PCCs) and mutual rank (MR) for gene co-expression (Obayashi and Kinoshita, 2009) were used to construct the GmELF4 network. A table including the top 50 genes with the greatest MR values was

\footnotetext{
${ }^{9}$ http://bioinformatics.cau.edu.cn/SFGD/
} 


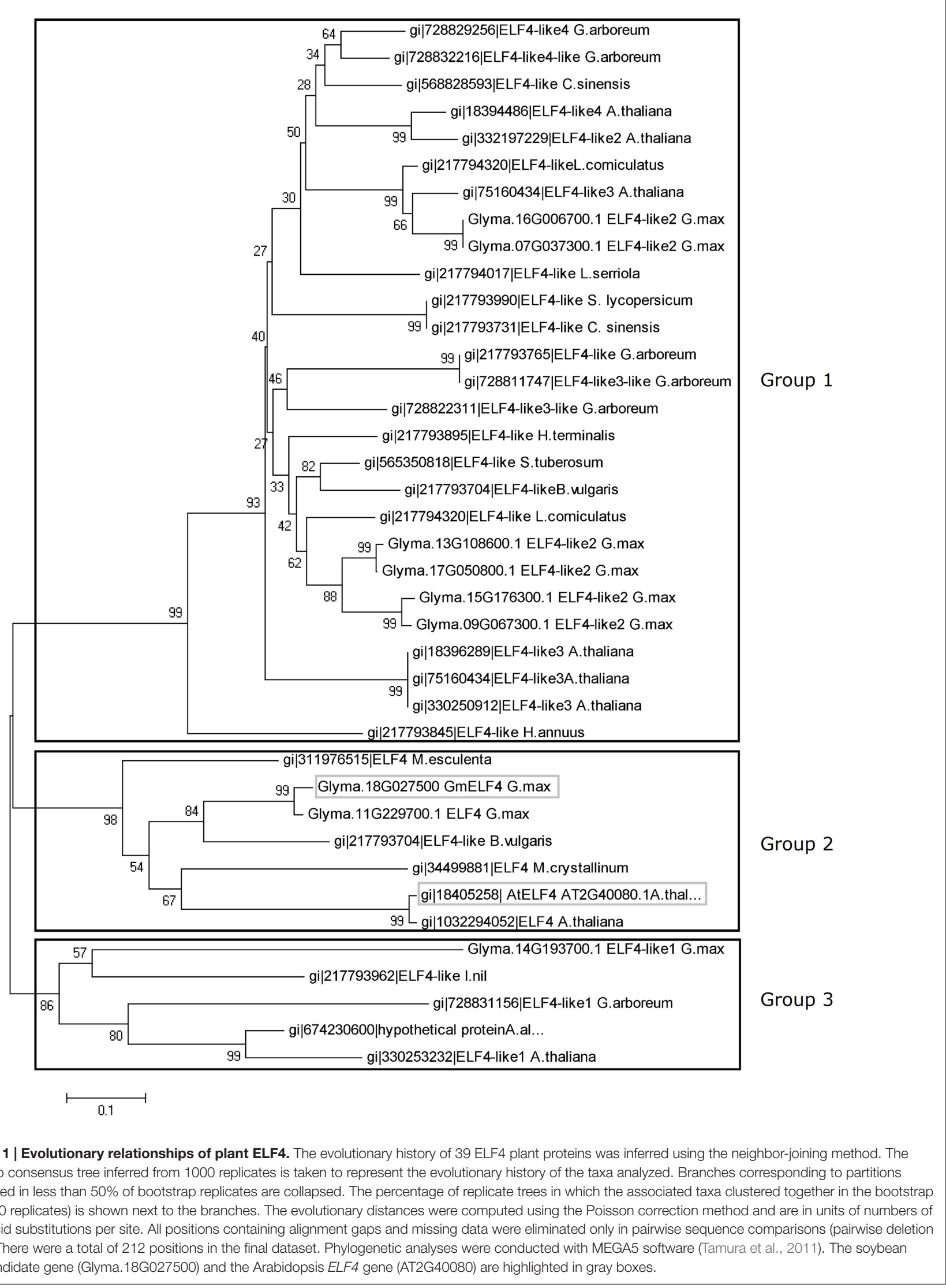




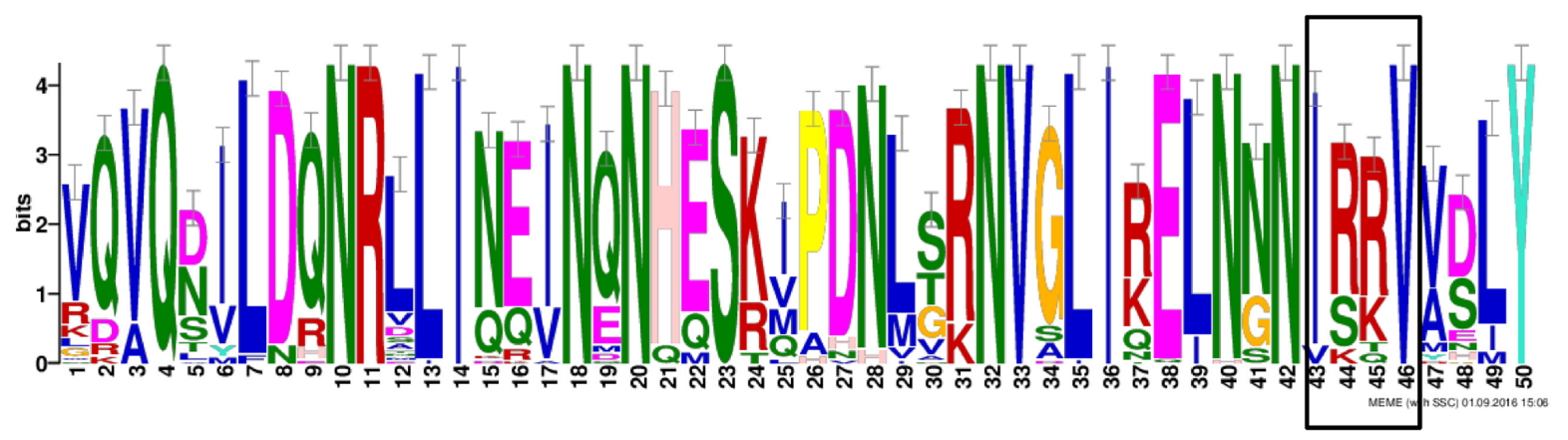

FIGURE 2 | Conserved amino acid motifs of the DUF1313 domain in ELF4 proteins in plants. Abscissa indicates amino acid residue number, and vertical axis indicates residue height, as described by the percentage of residues in the type. The box indicates the four conserved amino acid residues of the IRRV-type proteins from the DUF1313 family.
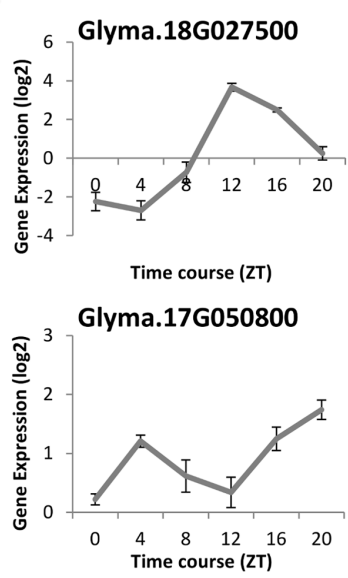
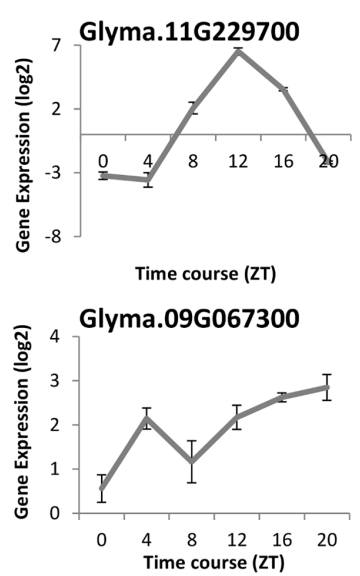
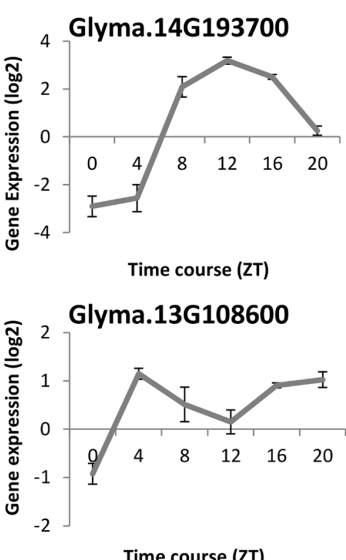
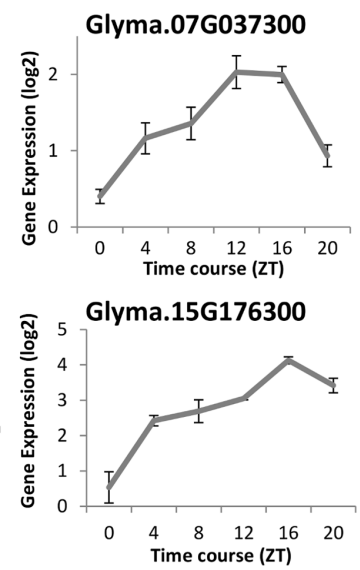

FIGURE 3 | RNA-Seq data of the soybean ELF4 homologous genes. Gene expression was measured in leaf tissues of BR16 soybeans subjected to short day (SD) conditions. Gene expression (Exp. Log. [ $\left.\log _{2}\right]$ ) was evaluated over a 24-h time course from the time the light came on (ZT0), in 4-h intervals. Error bars represent standard error (SEM).

constructed. The Cytoscape Web software program version 3.4.0 (Lopes et al., 2011) was used to display the GmELF4 (central node) and all its co-expression genes, sorted according to MR values.

\section{RESULTS}

\section{Identification and Characterization of ELF4 Genes in Soybean}

In this study, the soybean ELF4 candidate genes were identified using Arabidopsis ELF4 coding-sequences for in silico searches of the soybean genome. Nine GmELF4-like genes were identified (Glyma.07G037300, Glyma.09G067300, Glyma.18G027500, Glyma.11G229700, Glyma.13G108600, Glyma.14G193700, Glyma.15G176300, Glyma.16G006700, and Glyma.17G050800). To establish the relationships between the soybean ELF4 candidates and ELF4 proteins of Arabidopsis and other plants, we performed global alignments, followed by phylogenetic analysis and construction of a phylogenetic tree (Figure 1).
This tree indicates three large groups of ELF4 proteins. The soybean proteins encoded by GmELF4-like2 genes are gathered into group 1 with the Arabidopsis ELF4-like2, ELF4-like3, and ELF-like4 as well as with ELF4-like proteins from several other organisms. In group 2, the soybean ELF4 proteins encoded by Glyma.11G229700 and Glyma.18G027500 show close relation to the Arabidopsis ELF4 major protein (AT2G40080) (Figure 1). Group 3 constitutes the ELF4-like1 proteins from Arabidopsis, soybean and other plants (Figure 1).

Considering the importance of the functional domain for the classification and for investigating more deeply the putative function of the soybean candidates of ELF4, we performed a motif analysis of nine soybean ELF4 proteins together with 30 other plant ELF4 proteins (Figure 2). Our analysis revealed the presence of a DUF1313 motif conserved in all 39 ELF4 proteins, including the soybean ELF4 candidate genes Glyma.11G229700 and Glyma.18G027500. The Arabidopsis ELF 4 belongs to the DUF1313 family, whose members are divided into three major types, according to the substitution of four amino acid residues: IARV type, I(S/T/F)(K/R)V type and IRRV type, where the IRRV 

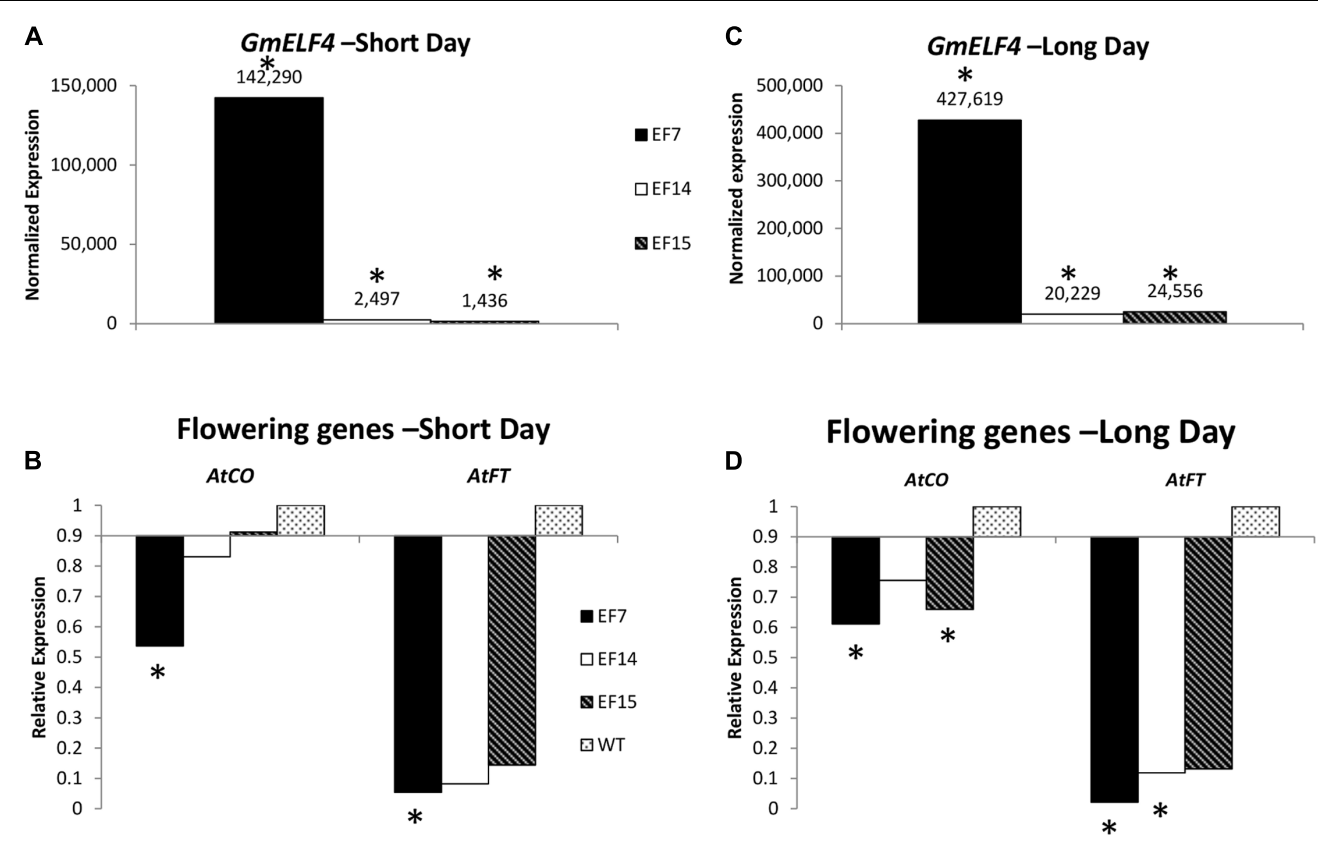

FIGURE 4 | Gene expression profile by RT-qPCR. The expression of GmELF4 (A) and of the flowering genes AtFT and AtCO (B) was evaluated in under short conditions. (C,D) Show the expression of GmELF4 and the flowering genes (AtFT and AtCO) under long day conditions. Leaves of the transgenic lines (EF7, EF14, and EF15) were evaluated at ZT8. Relative expression was calculated using the Rest2009 software package (Pfaffl et al., 2002) using the Arabidopsis endogenous gene PP2A for expression normalization. Wild-type (WT) plants expression was used for AtFT and AtCO expression calibration (B,D). Asterisks indicate statistically significant changes in gene expression, as calculated by Rest2009.

type is the largest (158 genes) (Li et al., 2015). Our analysis shows that the GmELF4 putative genes contain residues belonging to the IRRV type genes (Figure 2).

\section{GmELF4 Gene Expression in Soybeans}

To compare the diurnal oscillation of gene expression of ELF4 in soybeans and Arabidopsis, we evaluated the transcription levels of eight GmELF4 homologous genes by re-analyzing a validated RNA-Seq library published by Rodrigues et al. (2015). The expression profiles of the soybean ELF4 candidates were determined in V2-stage soybean plants under LD conditions (14 h light $/ 10 \mathrm{~h}$ dark) and temperature $\left(28^{\circ} \mathrm{C} / 20^{\circ} \mathrm{C}\right)$ cycles over a 24-h time course under well-watered conditions (Figure 3).

The mRNA levels of Glyma.18G027500, Glyma.11G229700, and Glyma.14G193700 peak at ZT12. The peak of transcripts of Glyma.07G037300 also occurs at ZT12, but, differently from the former genes, this peak is extended to ZT16. In contrast, Glyma.17G050800, Glyma.13G108600, and Glyma.09G067300 show two peaks of transcripts: one at ZT4 and another at ZT20. For instance, the Glyma.15G176300 expression level rises gradually throughout the day, peaking at ZT16 (Figure 3).

\section{Functional Characterization of a Soybean Putative ELF4 Homologous Gene by Overexpression in Arabidopsis \\ Plants}

To functionally characterize a soybean ELF4 homolog (GmELF4), we transformed Arabidopsis plants to overexpress constitutively the CDS of Glyma.18G027500. This soybean ELF4 homologous gene was selected due its resemblance to the features of AtELF4 at the phylogenetic and transcriptomic levels: the protein sequence of Glyma.18G027500 is very closely related to that of AtELF4 (Figure 1), and its transcripts oscillate throughout the day with the same profile of the AtELF4 transcripts (Figure 3).

\section{Molecular Characterization of Transgene Expression in Arabidopsis GmELF4-ox}

To evaluate the expression of GmELF4 in the three independent GmELF4-ox lines (EF7, EF14, and EF15), we analyzed the transcript levels of GmELF4 in leaves of transgenic versus WT plants cultivated under LD and SD conditions (Figures 4A,C). Our gene expression data show that GmELF4 was highly expressed in line EF7 for both SD and LD, while lines EF14 and EF15 had lower transcript levels (Figures 4A,C). The transgene expression was also detected by GFP fluorescence emission in leaves and roots of transgenic lines (Supplementary Figure 1).

\section{Transgenic Plants Phenotypes}

The genetic transformation of the three independent lines overexpressing the putative GmELF4 (GmELF4-ox), named EF7, EF14 and EF15, was confirmed by PCR using primers specific to the GmELF4 CDS (Supplementary Figure 2). The most striking phenotype of the transgenic GmELF4-ox lines compared to WT plants was flowering delay. Transformed Arabidopsis plants cultivated in both flowering induction conditions (LD) and noninductive conditions (SD) displayed a later transition from the vegetative to reproductive developmental phase (Figure 5 and 


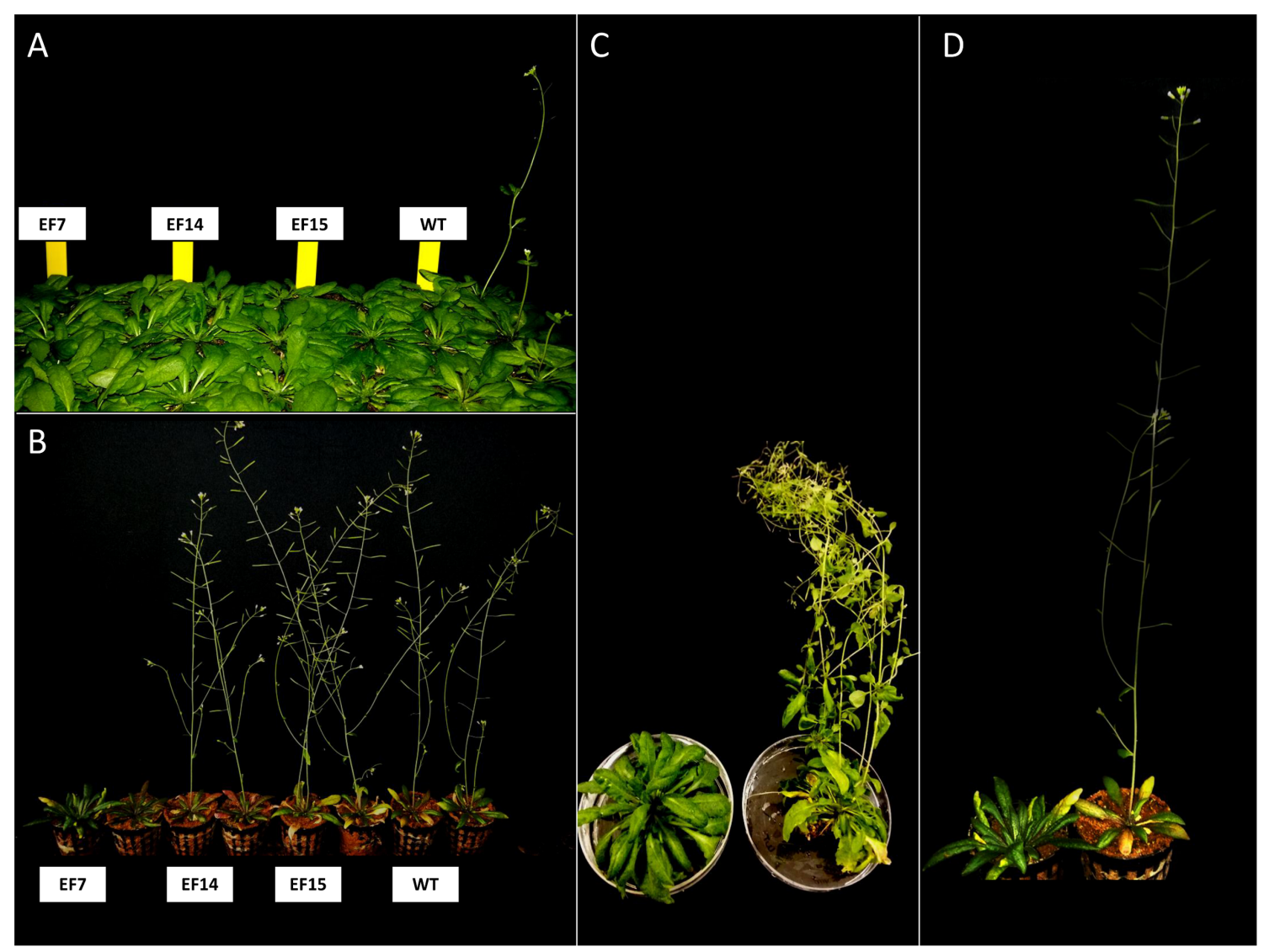

FIGURE 5 | Flowering in transgenic Arabidopsis plants. Transgenic (lines EF7, EF14, and EF15) and WT plants were grown under short-day (SD) and long-day (LD) with 10 and $16 \mathrm{~h}$ of light, respectively. Representatives of the earliest and latest flowering plants are shown for each genotype: (A) 8 week-old plants cultivated under SD; (B) 6 week-old plants cultivated under LD conditions. The contrasting flowering phenotypes of transgenic line EF7 and WT grown under SD (C) and LD (D) conditions are shown in detail: (C) 12 week-old plants cultivated under SD; (D) 6 week-old plants cultivated under LD.

Supplementary Figures 3, 4). Interestingly, the EF7 line displayed the most delayed flowering phenotype in response to both photoperiods, while WT plants were the first to flower (Figure 5 and Supplementary Figures 3, 4).

Another interesting feature of transgenic plants was the higher number of rosette leaves compared to WT plants, especially in transgenic line EF7 (Figure 6). This transgenic line had more leaves at the onset of the developmental transition from the vegetative to reproductive phase (Figure 6A), resulting in higher biomass accumulation (Figure 6B).

\section{Molecular Characterization of Key Flowering Genes in GmELF4-ox Arabidopsis}

The notable flowering delay of GmELF4-ox Arabidopsis encouraged us to investigate the molecular mechanisms behind the observed phenotypes.

To evaluate the impact of the overexpression of the soybean ELF4 homologous gene (Glyma.18G027500) on the expression of the key Arabidopsis flowering genes AtFT and AtCO, we analyzed transcript levels in transgenic plants compared to WT plants. Interestingly, our data reveal that the overexpression of GmELF4 had a significant inverse effect of impact on the levels of AtFT and AtCO transcripts, especially in the EF7 line, where the high levels of GmELF4 (Figures 4A,C) caused a substantial reduction in expression of AtFT and AtCO (Figures 4B,D). Similarly, lines EF14 and EF15 also presented reduced levels of AtFT and AtCO compared to WT plants, albeit in a slighter manner (Figures 4B,D).

\section{The Role of GmELF4 in Soybeans: Network Genes}

To better understand the function of the putative GmELF4 (Glyma.18G027500) in soybeans, we retrieved functionally related genes by constructing a co-expressed gene network using the SFGD database. The top 50 co-expressed genes from the GmELF4 network are listed in Table 1. A graphical representation of this network (Supplementary Figure 5) shows GmELF4 at the core of a network composed of genes that connect directly or indirectly to the core gene. Among those genes are several homologes of the circadian clock genes, including the GmELF4 paralog Glyma.11G229700, JUMONJI (Glyma.12G055000), CO-like 
(Glyma.02G223700, Glyma.16G050900, and Glyma.14G190400), and EARLY FLOWERING 3 (Glyma.04G050200) as well as the gene encoding GIGANTEA (GI) (Glyma.16G163200) (Table 1).

Interestingly, some of the GmELF4 network genes encode proteins related to abiotic stress responses, such as DnaJ chaperones (Glyma.07G197600 and Glyma.12G130000), chaperonins (Glyma.15G111400), a cold regulated protein (Glyma.17G242100) and a heat shock protein (Glyma. 02G288700) (Table 1).

\section{DISCUSSION}

\section{The ELF4 Putative Genes in Soybean}

ELF4 is a circadian clock gene essential for the maintenance of circadian rhythms and control of flowering in Arabidopsis. A previous study identified five ELF4-like genes in Arabidopsis: ELF4, ELF4-like1, ELF4-like2, ELF4-like3, and ELF4-like4 (Khanna et al., 2003). Here we identified a greater number of ELF4-like genes in soybean (nine), which agrees with a previous analysis of the soybean genome that shows the presence of complex circadian clock circuitry, generally composed of a greater number of components than that in Arabidopsis (Jung et al., 2012; Marcolino-Gomes et al., 2014; Rodrigues et al., 2015). Interestingly, all the soybean ELF4 candidates have a DUF1313 motif (IRRV-type) (Figure 2), a highly conserved domain exclusively found in plants, characteristic of ELF4 proteins ( $\mathrm{Li}$ et al., 2015), supporting the function of the soybean candidates to ELF4 genes/proteins in soybean.

The re-evaluation of a validated RNA-seq library (Rodrigues et al., 2015) allowed us to assess novel data regards the gene expression of the ELF4 soybean homologes along the day. These data reveals that the mRNA levels of Glyma.18G027500, Glyma.11G229700, and Glyma.14G193700 peak at ZT12 (Figure 3), in a similar manner as the Arabidopsis ELF4 gene (Doyle et al., 2002). This expression profile was validated in Glyma.18G027500 by RT-qPCR in a previous study (MarcolinoGomes et al., 2014). Interestingly, other GmELF4 genes showed diverse expression patterns throughout the day (Figure 3).

It is known that the accurate oscillation of the transcript levels of the circadian components throughout the day is crucial for accuracy of the circadian rhythms. The Arabidopsis ELF4 transcripts peak at specific periods allows it to interact with other circadian components to guarantee circadian precision and normal clock function (McWatters et al., 2007; Herrero et al., 2012). Thus, how can the differences found in the mRNA peaks observed for the different ELF4 genes from soybean be explained? The answer for this question may reside within the evolutionary history of soybean. The current soybean genome is the result of three rounds of polyploidy: (i) a genome triplication before the origin of the rosids ( $\sim 130$ to 240 million years ago), shared with grape, poplar and Arabidopsis; (ii) a genome duplication early in the legumes ( $\sim 58$ million years ago); and (iii) a duplication in the Glycine lineage $(\sim 13$ million years ago) (Severin et al., 2011).
An important feature of duplication is that it produces genetic redundancy, and a duplicated locus creates the opportunity for duplicates to explore new evolutionary functions (Flagel and Wendel, 2009). In a previous study of flowering genes in soybean, it was postulated that the differential accumulation of gene copies between soybean and Arabidopsis would be a possible evolutionary innovation that distinguishes the two species from one another (Jung et al., 2012). In addition, it has been verified that, if given sufficient time, one copy of a duplicate pair of genes can accumulate mutations that result in function divergence, or 'neofunctionalization' (Flagel and Wendel, 2009). The phylogenetic analyses of Arabidopsis and other angiosperms ELF4 genes reveals a complex gene history of duplication and loss within the ELF4 family (Kolmos et al., 2009). In this context, the different gene expression patterns observed for the ELF4 genes in soybean may be a reflex of functional divergence due modifications that occurred in this group of genes during the dynamic evolutionary history of the soybean genome. This divergence is possible because the role of sustaining the circadian oscillator, played by ELF4, can be fulfilled by the genes that oscillate to peak levels at ZT12 (such as Glyma.11G229700 and Glyma.18G027500), while the other paraloges may assume additional roles in the plant molecular circuitry. Furthermore, this mechanism may contribute to the evolution the circadian clock complexity of plants.

\section{Functional Characterization GmELF4 Homologous in Transgenic Arabidopsis}

The close phylogenetic relation between Glyma.18G027500 and the Arabidopsis ELF4 major protein (AT2G40080) (Figure 1), together with the similar expression patters between these ortholog genes (Figure 3) encouraged us to investigate the function of Glyma.18G027500 as a putative GmELF4 gene. The quantitation of the transcript levels of the putative GmELF4 in transgenic versus WT plants confirmed that the transgene was highly expressed in line EF7 for both SD and LD, while lines EF14 and EF15 had lower transcript levels (Figures 4A,C).

It is known that gene activity is not exclusively determined by the promoter that controls transcription (in our case, the CaMV35S promoter): epigenetic mechanisms also influence expression levels by blocking gene transcription or inhibiting mRNA accumulation. Thus, the difference of the transgene expression among lines EF7, EF14, and EF15 (Figures 4A,C) can be attributed to several factors including DNA methylation, production of aberrant RNAs, ectopic DNA-DNA interactions and the effect of the locus where the transgene was inserted (Stam et al., 1997; Butaye et al., 2005).

The most striking phenotype of the transgenic GmELF4$o x$ lines compared to WT plants was flowering delay. Similarly, McWatters et al. (2007) reported that the constitutive overexpression of the endogenous ELF4 gene in Arabidopsis resulted in flowering delay under inductive (LD) photoperiods, whereas under non-inductive conditions (SD) plants showed no additional delay in flowering. According to these authors, this phenotype resulted from the role of ELF4 as a floral repressor that coordinates floral transition as part of the 

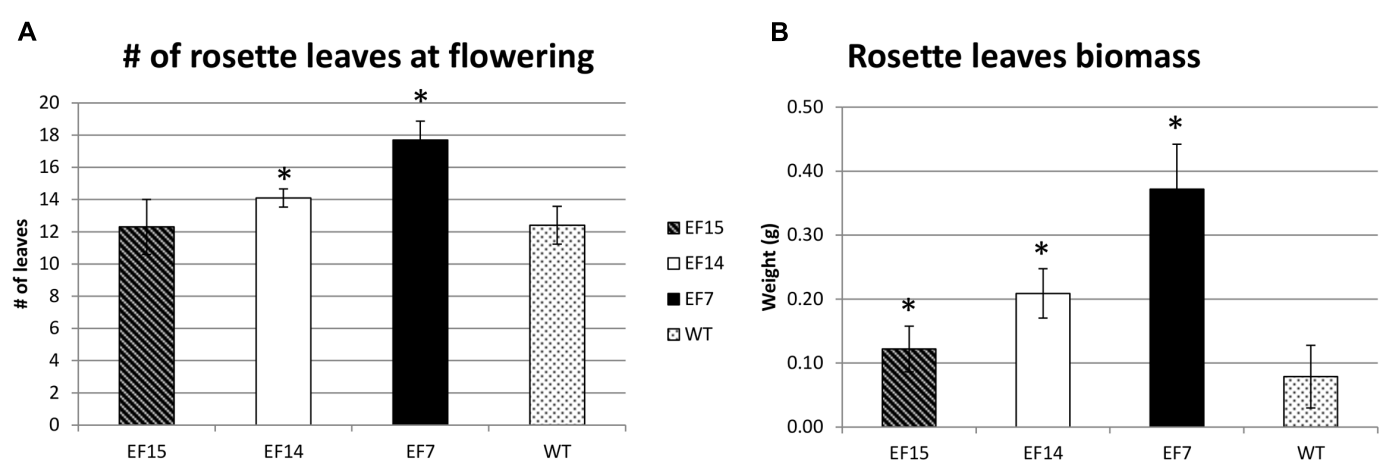

FIGURE 6 | Rosette leaves during development. The number of rosette leaves at beginning of flower (A), and the Rosette leaves biomass (B) are presented for transgenic lines (EF7, EF14, and EF15) and WT plants cultivated under $16 \mathrm{~h}$ of light (LD). The rosette leaf numbers were recorded when the primary inflorescence stem reach to $1 \mathrm{~cm}$. Mean values from 10 biological replicates are presented; error bars represent the standard deviation. Asterisks indicate statistical differences between the transgenic lines and WT values (Student's $t$-test $p<0.05$ ).

photoperiod pathway. Our data reveal that the soybean ELF4 homologous gene (Glyma.18G027500) is capable of delay Arabidopsis flowering, suggesting the molecular function of GmELF4 as a flowering repressor. Nonetheless, differently from the AtELF4 gene, the soybean homolog is also able to produce altered phenotypes (flowering delay) in SD conditions (Figures 5A,C and Supplementary Figure 4), indicating that this gene interacts differently with the Arabidopsis flowering control network.

In addition, the transgenic plants also presented a higher number of rosette leaves compared to WT plants (Figure 6A). This phenotype is likely a consequence of the flowering delay caused by GmELF4 overexpression, which allowed the plants to invest more in vegetative development, resulting in higher biomass accumulation (Figure 6B). Previous analysis of flowering control and biomass yield in Medicago truncatula mutants reveals that delayed flowering genotypes produce double the amount of biomass, with less lignin, compared to WT plants, showing that delaying floral initiation could be employed as a convenient biotechnology tool to improve simultaneously biomass quantity and quality in plants (Tadege et al., 2015). In this context, the GmELF4-ox phenotypes obtained in this study have great potential for the improvement of crops, such as legume forages, grasses and sugarcane, where flowering delay and improvement of biomass accumulation constitute the main goal.

\section{GmELF4 Alters the Expression of Key Arabidopsis Flowering Genes}

In Arabidopsis, flowering occurs due the action of the FLOWERING LOCUS T (FT) protein, and the amount of FT transcript is directly induced by the transcriptional activator $\mathrm{CO}$ protein, which is controlled by the circadian clock and light signaling (Kim et al., 2012, 2013). In turn, the AtELF4 protein is able to bind to the GI protein and target it to subnuclear compartments in the nucleus, sequestering GI from the $C O$ promoter, negatively affecting $C O$ expression levels and decreasing the amount of FT transcript, which results in absence of flower induction (Kim et al., 2012, 2013). Interestingly, our data evidence that the overexpression of GmELF4 caused a substantial reduction in expression of AtFT and AtCO (Figures 4B,D).

Our phenotypic and molecular data suggest that GmELF4 may play the role of its homologous gene (AtELF4) in reducing the expression of $C O$ and FT, which results in flowering delay. Further analysis should be carried on to confirm the ability of GmELF4 in sequestering GI from the CO promoter.

A previous study showed that the overexpression of an ELF4 gene from Doritaenopsis Happy Smile $\times$ Happy Valentine (DhEFL4) alters transgenic Arabidopsis phenotypes by postponing flowering by $12-14$ days, indicating that the function of ELF4 is highly conserved among plants (Chen et al., 2015). Our data shows similar phenotypic results for the GmELF4 gene, which have not been previously characterized, and our study also provides unpublished data regarding the molecular mechanisms behind these phenotypes.

Structural analysis of the ELF4 protein reveals that it forms a tight homodimer with $\alpha$-helical composition, critical for the protein function (Kolmos et al., 2009). Thus, studies about the occurrence of this structure in GmELF4 would contribute to a deeper understanding of this protein function. Furthermore, the capacity of the GmELF4 to form dimers could be extended to the formation of a complex with the AtELF4 protein, allowing this complex to act synergistically to generate the delayed flowering phenotypes observed in our transgenic lines.

\section{The GmELF4 Network Genes}

According to previous studies, the function of every gene is believed to rely on that of another gene(s), and together these genes form complex and intricate networks. Thus, gene co-expression databases are useful resources for predicting gene networks, because co-expressed genes are generally expected to be involved in related cellular functions (Obayashi and Kinoshita, 2009; Obayashi et al., 2009). These analyses show that GmELF4 network is composed by several homologes of the circadian clock and flowering genes (Table 1 and Supplementary Figure 5), 
TABLE 1 | Top 50 GmELF4 network genes.

\begin{tabular}{|c|c|c|c|c|}
\hline $\mathbf{M R}^{1}$ & $\mathrm{PCC}^{2}$ & G. $\max v 1.0^{3}$ & G. $\max W m 82 . a 2 . v 1^{4}$ & Annotation $^{5}$ \\
\hline 1.0 & 0.899 & Glyma11g35270 & Glyma.11G229700 & EARLY FLOWERING 4-like \\
\hline 5.5 & 0.729 & Glyma12g05890 & Glyma.12G055000 & JUMONJI transcription factor \\
\hline 6.9 & 0.764 & Glyma17g36060 & Glyma.17G242100 & COLD REGULATED PROTEIN 27 \\
\hline 7.6 & 0.768 & Glyma20g03910 & Glyma.20G031200 & Unknown protein \\
\hline 11.6 & 0.680 & Glyma02g38870 & Glyma.02G223700 & C2C2 (Zn) CO-like transcription factor \\
\hline 12.7 & 0.624 & Glyma18g46020 & Glyma.18G226200 & METHYLTRANSFERASE \\
\hline 14.4 & 0.730 & Glyma02g45810 & Glyma.02G288700 & HEAT SHOCK PROTEIN 42 \\
\hline 19.6 & 0.672 & Glyma11g19500 & No mapping & \\
\hline 20.0 & 0.694 & Glyma09g07240 & Glyma.16G163200 & PROTEIN GIGANTEA \\
\hline 20.5 & 0.696 & Glyma06g35550 & Glyma.06G235100 & Protein of unknown function (DUF3082) \\
\hline 21.4 & 0.693 & Glyma14g02970 & Glyma.14G026100 & HEAT SHOCK PROTEIN 42 \\
\hline 23.7 & 0.520 & Glyma04g05280 & Glyma.04G050200 & Protein EARLY FLOWERING 3 \\
\hline 24.3 & 0.676 & Glyma18g20600 & Glyma.18G142500 & Alpha-glucan, water dikinase/starch-related R1 protein \\
\hline 26.5 & 0.515 & Glyma16g05540 & Glyma.16G050900 & ZINC FINGER PROTEIN CONSTANS-LIKE 14-RELATED \\
\hline 26.7 & 0.642 & Glyma18g05060 & Glyma.18G044300 & ANKYRIN REPEAT DOMAIN-CONTAINING PROTEIN EMB506 \\
\hline 28.6 & 0.466 & Glyma14g36930 & Glyma.14G190400 & C2C2 (Zn) CO-like transcription factor \\
\hline 32.0 & 0.665 & Glyma02g15370 & Glyma.02G136000 & Ent-kaurene synthase/Ent-kaurene synthetase B \\
\hline 36.1 & 0.604 & Glyma05g29200 & Glyma.05G159400 & SHAGGY-RELATED PROTEIN KINASE ETA \\
\hline 37.1 & 0.603 & Glyma02g45280 & Glyma.02G283500 & HXXXD-TYPE ACYL-TRANSFERASE-RELATED \\
\hline 37.4 & 0.494 & Glyma20g33810 & Glyma.20G196000 & GLUCOSYL/GLUCURONOSYL TRANSFERASES \\
\hline 40.5 & 0.618 & Glyma17g12800 & No mapping & \\
\hline 46.3 & 0.640 & Glyma07g01400 & Glyma.07G011700 & PHOSPHATASE, ORPHAN 1, 2 \\
\hline 46.4 & 0.631 & Glyma18g07520 & Glyma.18G067500 & Similar to maternal effect embryo arrest 5 \\
\hline 53.6 & 0.577 & Glyma02g46870 & Glyma.02G299100 & SERINE ACETYLTRANSFERASE 1, CHLOROPLASTIC-RELATED \\
\hline 54.7 & 0.608 & Glyma08g45150 & Glyma.08G334500 & Similar to maternal effect embryo arrest 5 \\
\hline 56.9 & 0.524 & Glyma12g04750 & No mapping & \\
\hline 59.8 & 0.673 & Glyma07g31740 & Glyma.07G197600 & Molecular chaperone (DnaJ superfamily) \\
\hline 60.9 & 0.572 & Glyma17g13780 & Glyma.17G128500 & MYB/HD-like transcription factor \\
\hline 61.9 & 0.556 & Glyma16g33060 & Glyma.16G205500 & APO PROTEIN 1, CHLOROPLASTIC \\
\hline 62.4 & 0.551 & Glyma11g15600 & Glyma.U034800 & Unknown protein \\
\hline 66.3 & 0.566 & Glyma13g40850 & Glyma.13G333300 & METHYL-CPG-BINDING DOMAIN-CONTAINING PROTEIN 1-RELATED \\
\hline 75.9 & 0.527 & Glyma06g13190 & Glyma.06G126500 & PROTEIN DHS-1 \\
\hline 78.4 & 0.529 & Glyma17g18010 & Glyma.17G165200 & METAL TRANSPORTER NRAMP2-RELATED \\
\hline 83.0 & 0.634 & Glyma12g15560 & Glyma.12G130000 & DNAJ HOMOLOG DNJ-5 \\
\hline 83.9 & 0.620 & Glyma08g12370 & Glyma.08G117200 & SHAGGY-RELATED PROTEIN KINASE ETA \\
\hline 85.9 & 0.671 & Glyma16g26900 & Glyma.16G153000 & NDH-DEPENDENT CYCLIC ELECTRON FLOW 5 \\
\hline 86.4 & 0.456 & Glyma06g24420 & No mapping & \\
\hline 90.0 & 0.503 & Glyma13g31600 & No mapping & \\
\hline 91.6 & 0.576 & Glyma03g03590 & Glyma.03G030600 & CYTOCHROME P450 71B21-RELATED \\
\hline 93.2 & 0.573 & Glyma06g13450 & Glyma.06G129100 & ATP-DEPENDENT CLP PROTEASE REGULATORY SUBUNIT CLPX \\
\hline 100 & 0.466 & Glyma18g14490 & No mapping & \\
\hline 105 & 0.541 & Glyma06g20830 & Glyma.06G193800 & GIBBERELLIN-REGULATED PROTEIN 12-RELATED \\
\hline 105 & 0.620 & Glyma11g21670 & Glyma.11G155300 & Transglutaminase-like superfamily (Transglut_core2) \\
\hline 107 & 0.559 & Glyma03g03630 & Glyma.03G030800 & CYTOCHROME P450 71B21-RELATED \\
\hline 108 & 0.655 & Glyma08g39110 & Glyma.08G283700 & Alpha-glucan, water dikinase/starch-related R1 protein \\
\hline 109 & 0.652 & Glyma16g29640 & Glyma.16G177800 & Protein of unknown function (DUF3464) (DUF3464) \\
\hline 111 & 0.592 & Glyma15g11800 & Glyma.15G111400 & CHAPERONIN-LIKE RBCX PROTEIN \\
\hline 119 & 0.479 & Glyma18g51380 & Glyma.18G278800 & S-ADENOSYLMETHIONINE DECARBOXYLASE PROENZYME-RELATED \\
\hline 119 & 0.456 & Glyma15g40940 & Glyma.15G257600 & Deacetoxyvindoline 4-hydroxylase/desacetyoxyvindoline-17-hydroxylase \\
\hline 120 & 0.438 & Glyma08g22370 & Glyma.08G209300 & Unknown protein \\
\hline
\end{tabular}

${ }^{1}$ Mutual rank (MR) for gene co-expression (Obayashi and Kinoshita, 2009);

${ }^{2}$ Pearson's correlation coefficients (PCCs);

${ }^{3}$ Genemodel at the soybean genome version 1.0 (http://phytozome.net/soybean);

${ }^{4}$ Genemodel at the soybean genome version Wm82.a2.v14 (http://phytozome.net/soybean]);

${ }^{5}$ Gene annotation at the Phytozome database (http://phytozome.net/soybean). 
supporting the participation of GmELF4 as a component of the soybean circadian clock and flowering genes. Functional studies about the AtELF4 gene reveals that it functions as a small dimer, and its action is critical for buffering perturbations to the circadian clock evening-loop (Kolmos et al., 2009), a function that may be shared by the GmELF4 homolog.

Curiously, we also identified many abiotic stress responsive genes composing the GmELF4 network (Table 1 and Supplementary Figure 5). Several studies have shown connections between the plant responses to abiotic stresses (e.g., heat, cold, and drought) and the circadian clock (Fowler et al., 2005; Bieniawska et al., 2008; Legnaioli et al., 2009; Wilkins et al., 2010; Marcolino-Gomes et al., 2014; Rodrigues et al., 2015), suggesting a close connection between both pathways. In a previous study, we showed that severe drought stress imposition strongly represses the expression of GmELF4 in soybean (MarcolinoGomes et al., 2014). Together, this evidences that GmELF4 contributes to multiple important plant physiological responses, including the circadian clock, flowering control and responses to abiotic stresses, such as drought. The drought escape strategy entails the completion of the lifecycle in advance of the effects of drought and is a common strategy among annual plants, such as soybean; in Brassica rapa, for example, drought escape by early flowering has been proven to be an important adaptive mechanism acquired during plant evolution (Franks, 2011).

Based on our data, we suggest that GmELF4 may acts as a key negative flowering controller in soybean plants, in the same manner it does in transgenic Arabidopsis plants. Furthermore, its repression in response to drought stress may be part of a mechanism to promote early flowering to escape drought. Additional analyses in transgenic soybean plants (GmELF4-ox and/or elf mutants) are encouraged to provide additional insights about the flowering control mechanisms in this organism.

\section{CONCLUSION}

Here, we identified and functionally characterized a negative controller of flowering in soybeans (GmELF4) by overexpressing the CDS of this gene in Arabidopsis. The transference of knowledge between model and crop species will contribute to the understanding of the mechanisms underlying several aspects of plant physiology, including flowering. In addition to the contribution to the scientific understanding of the soybean flowering mechanisms, these findings also may contribute to the advance of soybean breeding programs that involve photoperiodic flowering induction, which impacts crop

\section{REFERENCES}

Adams, S., and Carré, I. A. (2011). Downstream of the plant circadian clock: output pathways for the control of physiology and development. Essays Biochem. 49, 53-69. doi: 10.1042/bse0490053

Alabadí, D., Oyama, T., Yanovsky, M. J., Harmon, F. G., Más, P., and Kay, S. A. (2001). Reciprocal regulation between TOC1 and LHY/CCA1 within the Arabidopsis circadian clock. Science 293, 880-883. doi: 10.1126/science.1061320 latitudinal and seasonal establishment as well as productivity. In addition, flowering repression phenotypes may be of great interest for crops such as legume forages, grasses, sugarcane and energy cane, where the main feature is biomass accumulation rather than seed production. Altogether, our results may help unveil a new perspective of photoperiodic flowering control in soybean. Future studies are encouraged to confirm the function of GmELF4 as a molecular hub connecting multiple physiological responses including the circadian clock, flowering control and responses to abiotic stresses.

\section{AUTHOR CONTRIBUTIONS}

JM-G and AN: contributed to the conception of the work, data acquisition, analysis, and interpretation, drafting the work and final approval of the version to be published; TN, HM, and MB: contributed to data acquisition, analysis, and interpretation as well as manuscript review; LH, RF-P, and FH: contributed to data interpretation and critically revised the manuscript for important intellectual content. All Authors agreed to be accountable for all aspects of the work in ensuring that questions related to the accuracy or integrity of any part of the work were appropriately investigated and resolved.

\section{FUNDING}

JM-G (Process PDI \# 312433/2015-8) and RF-P (Process PDS \# $158600 / 2014-2$ ) were supported by grants from CNPq.

\section{ACKNOWLEDGMENTS}

We thank the Coordenação de Aperfeiçoamento de Pessoal de Nível Superior (CAPES) and Conselho Nacional de Desenvolvimento Científico e Tecnológico (CNPq) for the financial support and the Brazilian Agricultural Research Corporation (Embrapa Soybean), for providing the laboratory and greehouse facilities where this study was conducted.

\section{SUPPLEMENTARY MATERIAL}

The Supplementary Material for this article can be found online at: http://journal.frontiersin.org/article/10.3389/fpls.2017.00618/ full\#supplementary-material

Altschul, S. F., Gish, W., Pennsylvania, T., and Park, U. (1990). Basic local alignment search tool. J. Mol. Biol. 215, 403-410. doi: 10.1016/S0022-2836(05) 80360-2

Bieniawska, Z., Espinoza, C., Schlereth, A., Sulpice, R., Hincha, D. K., and Hannah, M. A. (2008). Disruption of the Arabidopsis circadian clock is responsible for extensive variation in the cold-responsive transcriptome. Plant Physiol. 147, 263-279. doi: 10.1104/pp.108. 118059 
Butaye, K. M. J., Cammue, B. P. A., Delauré, S. L., and De Bolle, M. F. C. (2005). Approaches to minimize variation of transgene expression in plants. Mol. Breed. 16, 79-91. doi: 10.1007/s11032-005-4929-9

Chen, W., Qin, Q., Zheng, Y., Wang, C., Wang, S., Zhou, M., et al. (2015). Overexpression of Doritaenopsis Hybrid EARLY FLOWERING 4-like4 Gene, DhEFL4, postpones flowering in transgenic Arabidopsis. Plant Mol. Biol. Report. 34, 103-117. doi: 10.1007/s11105-015-0899-1

Dixon, L. E., Knox, K., Kozma-Bognar, L., Southern, M. M., Pokhilko, A., and Millar, A. J. (2011). Temporal repression of core circadian genes is mediated through EARLY FLOWERING 3 in Arabidopsis. Curr. Biol. 21, 120-125. doi: $10.1016 /$ j.cub.2010.12.013

Doyle, M. R., Davis, S. J., Bastow, R. M., McWatters, H. G., Kozma-Bognár, L., Nagy, F., et al. (2002). The ELF4 gene controls circadian rhythms and flowering time in Arabidopsis thaliana. Nature 419, 74-77. doi: 10.1038/nature 00954

Fehr, W. R., Caviness, C. E., Burmood, D. T., and Pennington, J. S. (1971). Stage of development descriptions for soybeans, Glycine max (L.) Merrill1. Crop Sci. 11, 929. doi: 10.2135/cropsci1971.0011183X001100060051x

Flagel, L. E., and Wendel, J. F. (2009). Gene duplication and evolutionary novelty in plants. New Phytol. 183, 557-564. doi: 10.1111/j.1469-8137.2009. 02923.x

Fowler, S. G., Cook, D., and Thomashow, M. F. (2005). Low temperature induction of Arabidopsis CBF1, 2, and 3 is gated by the circadian clock. Plant Physiol. 137, 961-968. doi: 10.1104/pp.104.058354

Franks, S. J. (2011). Plasticity and evolution in drought avoidance and escape in the annual plant Brassica rapa. New Phytol. 190, 249-257. doi: 10.1111/j.1469-8137. 2010.03603.x

Gendron, J. M., Pruneda-Paz, J. L., Doherty, C. J., Gross, A. M., Kang, S. E., and Kay, S. A. (2012). Arabidopsis circadian clock protein, TOC1, is a DNAbinding transcription factor. Proc. Natl. Acad. Sci. U.S.A. 109, 3167-3172. doi: $10.1073 /$ pnas. 1200355109

Godfray, H. C. J., Beddington, J. R., Crute, I. R., Haddad, L., Lawrence, D., Muir, J. F., et al. (2010). Food security: the challenge of feeding 9 billion people. Science 327, 812-818. doi: $10.1126 /$ science. 1185383

Harmer, S. L., Hogenesch, J. B., Straume, M., Chang, H. S., Han, B., Zhu, T., et al. (2000). Orchestrated transcription of key pathways in Arabidopsis by the circadian clock. Science 290, 2110-2113. doi: 10.1126/science.290. 5499.2110

Harrison, S. J., Mott, E. K., Parsley, K., Aspinall, S., Gray, J. C., and Cottage, A. (2006). A rapid and robust method of identifying transformed Arabidopsis thaliana seedlings following floral dip transformation. Plant Methods 2:19. doi: 10.1186/1746-4811-2-19

Helfer, A., Nusinow, D. A., Chow, B. Y., Gehrke, A. R., Bulyk, M. L., and Kay, S. A. (2011). LUX ARRHYTHMO encodes a nighttime repressor of circadian gene expression in the Arabidopsis core clock. Curr. Biol. 21, 126-133. doi: 10.1016/j.cub.2010.12.021

Herrero, E., Kolmos, E., Bujdoso, N., Yuan, Y., Wang, M., Berns, M. C., et al. (2012). EARLY FLOWERING4 recruitment of EARLY FLOWERING3 in the nucleus sustains the Arabidopsis circadian clock. Plant Cell 24, 428-443. doi: $10.1105 /$ tpc. 111.093807

Ito, S., Song, Y. H., Josephson-Day, A. R., Miller, R. J., Breton, G., Olmstead, R. G., et al. (2012). FLOWERING BHLH transcriptional activators control expression of the photoperiodic flowering regulator CONSTANS in Arabidopsis. Proc. Natl. Acad. Sci. U.S.A. 109, 3582-3587. doi: 10.1073/pnas.11188 76109

Jang, S., Marchal, V., Panigrahi, K. C. S., Wenkel, S., Soppe, W., Deng, X.-W., et al. (2008). Arabidopsis COP1 shapes the temporal pattern of $\mathrm{CO}$ accumulation conferring a photoperiodic flowering response. EMBO J. 27, 1277-1288. doi: 10.1038/emboj.2008.68

Jung, C. H., Wong, C. E., Singh, M. B., and Bhalla, P. L. (2012). Comparative genomic analysis of soybean flowering genes. PLoS ONE 7:e38250. doi: 10.1371/ journal.pone. 0038250

Karimi, M., Inzé, D., and Depicker, A. (2002). GATEWAYTM vectors for Agrobacterium-mediated plant transformation. Trends Plant Sci. 7, 193-195. doi: 10.1016/S1360-1385(02)02251-3

Khanna, R., Kikis, E. A., and Quail, P. H. (2003). EARLY FLOWERING 4 functions in phytochrome B-regulated seedling De-etiolation. Plant Physiol. 133, 1530-1538. doi: 10.1104/pp.103.030007
Kikis, E. A., Khanna, R., and Quail, P. H. (2005). ELF4 is a phytochrome-regulated component of a negative-feedback loop involving the central oscillator components CCA1 and LHY. Plant J. 44, 300-313. doi: 10.1111/j.1365-313X. 2005.02531.x

Kim, Y., Lim, J., Yeom, M., Kim, H., Kim, J., Wang, L., et al. (2013). ELF4 regulates GIGANTEA chromatin access through subnuclear sequestration. Cell Rep. 3, 671-677. doi: 10.1016/j.celrep.2013.02.021

Kim, Y., Yeom, M., Kim, H., Lim, J., Koo, H. J., Hwang, D., et al. (2012). GIGANTEA and EARLY FLOWERING 4 in Arabidopsis exhibit differential phase-specific genetic influences over a diurnal cycle. Mol. Plant 5, 678-687. doi: $10.1093 / \mathrm{mp} / \mathrm{sss} 005$

Kolmos, E., Nowak, M., Werner, M., Fischer, K., Schwarz, G., Mathews, S., et al. (2009). Integrating ELF4 into the circadian system through combined structural and functional studies. HFSP J. 3, 350-366. doi: 10.2976/1.3218766

Kong, F., Liu, B., Xia, Z., Sato, S., Kim, B. M., Watanabe, S., et al. (2010). Two coordinately regulated homologs of FLOWERING LOCUS $\mathrm{T}$ are involved in the control of photoperiodic flowering in soybean. Plant Physiol. 154, 1220-1231. doi: 10.1104/pp.110.160796

Legnaioli, T., Cuevas, J., and Mas, P. (2009). TOC1 functions as a molecular switch connecting the circadian clock with plant responses to drought. EMBO J. 28, 3745-3757. doi: 10.1038/emboj.2009.297

Li, J., Hu, E., Chen, X., Xu, J., Lan, H., Li, C., et al. (2015). Evolution of DUF1313 family members across plant species and their association with maize photoperiod sensitivity. Genomics 107, 199-207. doi: 10.1016/j.ygeno.2016. 01.003

Lopes, C. T., Franz, M., Kazi, F., Donaldson, S. L., Morris, Q., Bader, G. D., et al. (2011). Cytoscape Web: an interactive web-based network browser. Bioinformatics 27, 2347-2348. doi: 10.1093/bioinformatics/btq430

Marcolino-Gomes, J., Rodrigues, F. A., Fuganti-Pagliarini, R., Bendix, C., Nakayama, T. J., Celaya, B., et al. (2014). Diurnal oscillations of soybean circadian clock and drought responsive genes. PLoS ONE 9:e86402. doi: 10. 1371/journal.pone.0086402

McClung, C. R. (2011). The genetics of plant clocks. Adv. Genet. 74, 105-139. doi: 10.1016/B978-0-12-387690-4.00004-0

McWatters, H. G., Kolmos, E., Hall, A., Doyle, M. R., Amasino, R. M., Gyula, P., et al. (2007). ELF4 is required for oscillatory properties of the circadian clock. Plant Physiol. 144, 391-401. doi: 10.1104/pp.107.096206

Nusinow, D. A., Helfer, A., Hamilton, E. E., King, J. J., Imaizumi, T., Schultz, T. F., et al. (2011). The ELF4-ELF3-LUX complex links the circadian clock to diurnal control of hypocotyl growth. Nature 475, 398-402. doi: 10.1038/nature10182

Obayashi, T., Hayashi, S., Saeki, M., Ohta, H., and Kinoshita, K. (2009). ATTEDII provides coexpressed gene networks for Arabidopsis. Nucleic Acids Res. 37, D987-D991. doi: 10.1093/nar/gkn807

Obayashi, T., and Kinoshita, K. (2009). Rank of correlation coefficient as a comparable measure for biological significance of gene coexpression. DNA Res. 16, 249-260. doi: 10.1093/dnares/dsp016

Pfaffl, M. W., Horgan, G. W., and Dempfle, L. (2002). Relative expression software tool (REST) for group-wise comparison and statistical analysis of relative expression results in real-time PCR. Nucleic Acids Res. 30, e36. doi: 10.1093/ nar/30.9.e36

Pokhilko, A., Fernández, A. P., Edwards, K. D., Southern, M. M., Halliday, K. J., and Millar, A. J. (2012). The clock gene circuit in Arabidopsis includes a repressilator with additional feedback loops. Mol. Syst. Biol. 8, 574. doi: 10.1038/msb.2012.6

Rodrigues, F. A., Fuganti-Pagliarini, R., Marcolino-Gomes, J., Nakayama, T. J., Molinari, H. B. C., Lobo, F. P., et al. (2015). Daytime soybean transcriptome fluctuations during water deficit stress. BMC Genomics 16:505. doi: 10.1186/ s12864-015-1731-x

Rozen, S., and Skaletsky, H. (1999). "Primer3 on the WWW for general users and for biologist programmers," in Bioinformatics Methods and Protocols Methods in Molecular Biology, eds S. Misener and S. A. Krawetz (New York City, NY: Humana Press), 365-386.

Sawa, M., Nusinow, D. A., Kay, S. A., and Imaizumi, T. (2007). FKF1 and GIGANTEA complex formation is required for day-length measurement in Arabidopsis. Science 318, 261-265. doi: 10.1126/science.1146994

Severin, A. J., Cannon, S. B., Graham, M. M., Grant, D., and Shoemaker, R. C. (2011). Changes in twelve homoeologous genomic regions in soybean following three rounds of polyploidy. Plant Cell 23, 3129-3136. doi: 10.1105/tpc.111. 089573 
Stam, M., Mol, J., and Kooter, J. (1997). The silence of genes in transgenic plants. Ann. Bot. 79, 3-12. doi: 10.1006/anbo.1996.0295

Tadege, M., Chen, F., Murray, J., Wen, J., Ratet, P., Udvardi, M. K., et al. (2015). Control of vegetative to reproductive phase transition improves biomass yield and simultaneously reduces lignin content in Medicago truncatula. Bioenergy Res. 8, 857-867. doi: 10.1007/s12155-014-9565-y

Tamura, K., Peterson, D., Peterson, N., Stecher, G., Nei, M., and Kumar, S. (2011). MEGA5: molecular evolutionary genetics analysis using maximum likelihood, evolutionary distance, and maximum parsimony methods. Mol. Biol. Evol. 28, 2731-2739. doi: 10.1093/molbev/msr121

Turnbull, C. (2011). Long-distance regulation of flowering time. J. Exp. Bot. 62, 4399-4413. doi: 10.1093/jxb/err 191

Watanabe, S., Harada, K., and Abe, J. (2012). Genetic and molecular bases of photoperiod responses of flowering in soybean. Breed. Sci. 61, 531-543. doi: 10.1270/jsbbs.61.531

Wenkel, S., Turck, F., Singer, K., Gissot, L., Le Gourrierec, J., Samach, A., et al. (2006). CONSTANS and the CCAAT box binding complex share a functionally important domain and interact to regulate flowering of Arabidopsis. Plant Cell 18, 2971-2984. doi: 10.1105/tpc.106.043299

Wilkins, O., Bräutigam, K., and Campbell, M. M. (2010). Time of day shapes Arabidopsis drought transcriptomes. Plant J. 63, 715-727. doi: 10.1111/j.1365313X.2010.04274.X

Wu, F., Price, B. W., Haider, W., Seufferheld, G., Nelson, R., and Hanzawa, Y. (2014). Functional and evolutionary characterization of the CONSTANS gene family in short-day photoperiodic flowering in soybean. PLoS ONE 9:e85754. doi: 10.1371/journal.pone.0085754
Yamaguchi, A., Kobayashi, Y., Goto, K., Abe, M., and Araki, T. (2005). TWIN SISTER of FT (TSF) acts as a floral pathway integrator redundantly with FT. Plant Cell Physiol. 46, 1175-1189. doi: 10.1093/pcp/pci151

Yerushalmi, S., and Green, R. M. (2009). Evidence for the adaptive significance of circadian rhythms. Ecol. Lett. 12, 970-981. doi: 10.1111/j.1461-0248.2009. 01343.x

Yu, J., Zhang, Z., Wei, J., Ling, Y., Xu, W., and Su, Z. (2014). SFGD: a comprehensive platform for mining functional information from soybean transcriptome data and its use in identifying acyl-lipid metabolism pathways. BMC Genomics 15:271. doi: 10.1186/1471-2164-15-271

Zhang, X., Henriques, R., Lin, S., Niu, Q.-W., and Chua, N.-H. (2006) Agrobacterium-mediated transformation of Arabidopsis thaliana using the floral dip method. Nat. Protoc. 1, 641-646. doi: 10.1038/nprot. 2006.97

Conflict of Interest Statement: The authors declare that the research was conducted in the absence of any commercial or financial relationships that could be construed as a potential conflict of interest.

Copyright (C) 2017 Marcolino-Gomes, Nakayama, Molinari, Basso, Henning, Fuganti-Pagliarini, Harmon and Nepomuceno. This is an open-access article distributed under the terms of the Creative Commons Attribution License (CC BY). The use, distribution or reproduction in other forums is permitted, provided the original author(s) or licensor are credited and that the original publication in this journal is cited, in accordance with accepted academic practice. No use, distribution or reproduction is permitted which does not comply with these terms. 